\title{
Ultraviolet Dust Grain Properties in Starburst Galaxies: Evidence from Radiative Transfer Modeling and Local Group Extinction Curves
}

\author{
Karl D. Gordon \\ Steward Observatory, University of Arizona, Tucson, AZ 85721, USA \\ email:kgordon@as.arizona.edu
}

\begin{abstract}
This paper summarizes the evidence of the ultraviolet properties of dust grains found in starburst galaxies. Observations of starburst galaxies clearly show that the $2175 \AA$ feature is weak or absent. This can be the result of radiative transfer effects (mixing the dust and stars) or due to dust grains which do not have this feature. Spherical DIRTY radiative transfer models imply that it is not radiative transfer effects, but other radiative transfer models with disk/bulge geometries have found cases where it could be radiative transfer effects. Recent work on the extinction curves in the Magellanic Clouds and Milky Way has revealed that the traditional explanation of low metallicity for the absence of the $2175 \AA$ f feature in the Small Magellanic Cloud is likely incorrect. The SMC has one sightline with a $2175 \AA$ feature and the Milky Way has sightlines without this feature. In addition, where the $2175 \AA$ feature is found to be weak or absent in both Magellanic Clouds and the Milky Way, there is evidence for recent star formation. Taking the sum of the radiative transfer modeling of starburst galaxies and the behavior of Local Group extinction curves, it is likely that the dust grains in starburst galaxies intrinsically lack the $2175 \AA$ feature.
\end{abstract}

\section{INTRODUCTION}

There are two main reasons to study the dust in galaxies where single stars are unresolvable with current telescopes.

1) To directly study the dust itself as galaxies provide a much wider range of environments than found in the Local Group. These different environments can be characterized by more extreme values of radiation field density, radiative field hardness, shock frequency, and initial conditions (eg., metallicity). Such different environments can significantly impact the formation and destruction of dust grains.

2) To more accurately account for the effects of dust on observations of the stars and gas present in galaxies. This accounting allows for the study of stars and gas in galaxies to be carried out more accurately.

In order to study dust in such galaxies, a model of how the mixing of dust with stars and gas affects to the observations of a galaxy is required. One such model is the DIRTY dust radiative transfer model [1, 2] which uses Monte Carlo techniques to compute the radiative transfer of photons through dust and self-consistently accounts for the dust re-emission in the infrared including equilibrium and non-equilibrium emission.

This paper concentrates on the ultraviolet dust grain properties in starburst galaxies determined using the DIRTY model and implied from extinction curves measured in 
the Local Group. The study of dust in galaxies is much larger than this, especially in light of the wealth of information available in the infrared where the dust grain emission dominated the spectral energy distribution of a galaxy. The connection between ultraviolet and infrared dust grain properties is not direct, but determined using dust grain models [3, 4, 5] which have their own problems. For example, I have found that using empirically determined dust scattering properties in the ultraviolet [6] is crucial to reproducing the ultraviolet colors of starburst galaxies.

\section{STARBURST GALAXIES}

Starburst galaxies provide ideal environments to probe dust grain properties in extreme environments as they are intrinsically bright in the ultraviolet where dust grain properties show large variation [7, 8, 9]. The environments probed by starbursts range from very metal poor (eg., I Zw 18) to very metal rich and most starbursts are likely characterized by high and hard radiation fields and elevated shock frequencies. Starbursts probe the more active environments of those possible in galaxies in general.

The study of the type of dust found in starburst galaxies really got going with the work of Calzetti et al. [10] and Calzetti [11]. In these studies, a variant of the standard pair method was used to derive an empirical attenuation curve appropriate for ultraviolet bright starburst galaxies. This empirical curve clearly lacked the strong $2175 \AA$ absorption feature seen in almost all known dust extinction curves. In addition, this curve was grayer than most dust extinction curves. The question was then: Is the lack of this $2175 \AA$ feature and the grayer curve due to different dust grain properties or radiative transfer effects? At the time, only a small number of sightlines in the Small Magellanic Cloud (SMC) were known to lack this feature [12]. This lack was attributed to the low metallicity of the SMC with the higher metallicities of the Large Magellanic Cloud and the Milky Way resulting in the ubiquity of the $2175 \AA$ feature in their dust [7, 8]. But, the starburst galaxies studied by Calzetti et al. [10] had metallicities spanning the known range from lower than the SMC to higher than the Milky Way. Radiative transfer effects were seen as the probable cause of the lack of the $2175 \AA$ feature. The complicated mixing of the dust, gas, and many stars in unresolved observations of galaxies implies that radiative transfer effects are important. Such mixing means that the stars in the observing beam have different dust columns and significant scattered flux is included in any measurement. As a result, the attenuation curve for a galaxy is not dependent only on dust grain properties, like that for the extinction curve towards a single resolved star in the Local Group, but is also dependent on the geometry of the dust, gas, and stars.

Motivated by the starburst results and improvements in the DIRTY dust radiative transfer model to include nonhomogeneous dust distributions [13], Gordon et al. [14] investigated the importance of radiative transfer effects on determining the shape of the empirical starburst attenuation curve. After investigating a number of dust/star geometries, they found that the lack of the $2175 \AA$ feature could not be explained by radiative transfer effects. The only way to remove the $2175 \AA$ feature from the attenuation curve was for the dust grains themselves to lack the feature. This result coupled with the large range in metallicities in the starburst galaxies implied that it was the starburst environ- 
ment which was responsible for the lack of the $2175 \AA$ feature. The dust grains were either newly formed without the feature or this feature was destroyed by the hard radiation field and elevated shock frequency in starbursts. Using the same or similar analysis, this result was extended to higher redshifts $(z \approx 3)$ by Gordon et al. [15] and Vijh et al. [16] where it was also found that most high-redshift (and likely starburst) galaxies lack a $2175 \AA$ bump. A detailed analysis of the Calzetti [11] attenuation curve by Witt and Gordon [17] found that a clumpy SHELL geometry with SMC dust, $\tau_{V} \sim 1.5$ DIRTY model best fit both the grayness of the curve and the lack of the $2175 \AA$ feature.

Since the work utilizing simple spherical DIRTY models, other studies have found ways to produce attenuation curves with weak or nearly absent $2175 \AA$ features while using dust grains with Milky Way-like dust (i.e., a strong $2175 \AA$ feature). Using the GRASIL code, Granato et al. [18] found that differentially embedding sources of different spectral types (basically by stellar lifetime) in molecular clouds in a disk/bulge geometry with clumpy structure and Milky Way-like dust resulted in attenuation curves with weak or absent $2175 \AA$ features. Fischera et al. [19] studied the attenuation curves produced by a foreground screen with a log-normal density distribution of Milky Waylike dust and was able to reproduce the grayness of the Calzetti [11] curve, but was only able to reduce the strength of the $2175 \AA$ feature. Finally, Pierini et al. [20] used DIRTY models of disk/bulge galaxies and found that for highly inclined, weak bulge galaxies the $2175 \AA$ feature was very weak in the global attenuation curves.

As a result of these studies [18, 19, 20], it is clear that there are dust/star geometries which can reproduce most, if not all, of the characteristics of the Calzetti [11] starburst curve. But it is not clear that the necessary geometries (disk/bulge or foreground screen) are appropriate for the starburst galaxies which are usually seen as nuclear dominated or have highly irregular morphologies.

\section{LOCAL GROUP EXTINCTION CURVES}

An important part of the type of dust we expect to find in galaxies is driven by our detailed knowledge of dust in the Local Group. One of the most powerful ways to study the properties of dust is to determine ultraviolet through near-infrared extinction curves towards single stars. As the dust measured by observing a star is distributed in a foreground screen, the derived extinction curve is only dependent on the properties of the dust grains (size, composition, and shape). With current telescopes and instruments, this work is limited to Local Group galaxies.

Prior to the work on starburst galaxies, it was thought that most galaxies would have dust with a $2175 \AA$ feature because all known sightlines in the Milky Way [7, 8] and the Large Magellanic Cloud [21, 22] had this feature. Only in the Small Magellanic Cloud was the $2175 \AA$ feature absent [12] and this was understood to be due to the low metallicity of this galaxy. The lack of the $2175 \AA$ feature in starburst galaxies with metallicities like those found in the Milky Way and LMC questioned the explanation for the SMC lacking the $2175 \AA$ feature. In fact, the variation in the LMC extinction curves between those near and far from the 30 Dor star forming region pointed to a different explanation [22]. It was found that the extinction curves near 30 Dor had weaker $2175 \AA$ 
features and somewhat steeper far-ultraviolet extinctions than those found in the rest of the LMC.

The existence of two explanations (low metallicity versus nearby active star formation) for the weakness or lack of a $2175 \AA$ feature motivated new work on extinction curves in the Local Group.

In the SMC, Gordon and Clayton [23] searched the IUE archives and found it was possible to generate accurate extinction curves for only four sightlines. Three of the four curves were basically linear with $1 / \lambda$ and had no detectable $2175 \AA$ feature. The fourth curve was much more like that found in the Milky Way with a $2175 \AA$ feature and weaker far-ultraviolet extinction compared to the rest of the SMC curves. This Milky Way-like curve was first published in Lequeux et al. [24] but dismissed by Prevot et al. [12] as anomalous. Gordon and Clayton [23] found that this Milky Way-like curve was located in a more quiescent region than the other three curves which were located in the star forming bar of the SMC. One additional sightline was added to the sample of SMC extinction curves by Gordon et al. [9] using new HST/STIS observations. This new sightline was in the star forming bar and was very similar to the previously known extinction curves in this same region. Thus, the spatial variations in the five known extinction curves in the SMC are consistent with the explanation that the lack of the $2175 \AA$ feature is due to nearby active star formation.

In the LMC, Misselt et al. [25] searched the IUE archives and was able to construct 19 accurate extinction curves from the available data. Like previous studies, this work found significant differences between those sightlines near 30 Dor and those in the rest of the LMC. Misselt et al. [25] found that this difference was caused by the sightlines associated with the supergiant shell LMC 2 near 30 Dor, not 30 Dor itself. Thus, they found very significant differences (especially in their $2175 \AA$ feature strengths) when the 19 sightlines were grouped between those associated with the LMC 2 shell and those in the rest of the LMC. Like the SMC, the spatial variation of the LMC extinction curves is consistent with active star formation being the cause.

In the Milky Way, the most comprehensive extinction curve study to date has been the recent work of Valencic et al. [26]. Like the studies in the SMC and LMC [23, 25], the IUE archive was searched and a total of 417 extinction curves created. The overwhelming majority of these curves $(93 \%)$ were well characterized by the Cardelli et al. [7] $R_{V}$ dependent relationship with only 4 curves showing systematic deviation from this relationship. HD 204827 is one of these 4 curves and a detailed study of this sightline [27] found that after subtracting a well measured foreground component, the remaining extinction curve was equivalent within the uncertainties to the extinction curves found in the SMC star forming bar (i.e., linear with $1 / \lambda$ and lacking a $2175 \AA$ bump). The local environment of HD 204827 has evidence for a recent supernova shock. In a study of 30 low reddening, long sightlines in the Milky Way, Clayton et al. [28] found that a subsample which had extinction curves like that found in the LMC 2 shell sample [25]. This subsample were all in the same region of the Milky Way and displayed $\mathrm{N}(\mathrm{Ca} \mathrm{II}) / \mathrm{N}(\mathrm{Na}$ I) ratios and velocities indicating recent dust destruction. Like the work in Magellanic Clouds, new studies in the Milky Way have revealed evidence that processing of dust can cause the weakening or disappearance of the $2175 \AA$ feature and strengthening of the far-ultraviolet extinction. 
Examining the sum of work on extinction curves in the Milky Way and Magellanic Clouds, it is clear that there is a continuum of dust extinction curves extending from those represented by the Cardelli et al. [7] relationship with strong $2175 \AA$ features to those like those found in the SMC bar with no detectable $2175 \AA$ feature [9]. This implies that it is more accurate to describe the Cardelli et al. [7] relationship as referring to dust in quiescent environments and SMC bar extinction curves as referring to dust in much more active environments. But even this picture of dust ultraviolet extinction curve variations is incomplete as dust in molecular clouds has not been well measured. Only the Taurus molecular cloud has been probed in the ultraviolet through the HD 29647 and HD 283809 sightlines [29]. By subtracting a foreground extinction curve measurement from these two sightlines, Whittet et al. [30] found that the $2175 \AA$ bump disappeared. This is like the dust found in the SMC bar, but the far-ultraviolet extinction in this dust was much weaker. This is good evidence that the true range of dust extinction curves likely includes another extreme, that found in molecular clouds.

\section{DISCUSSION}

In this paper, I have attempted to summarize work I have been involved in relating to the dust found in starburst galaxies and Local Group extinction curve work motivated in part by the starburst galaxy results. Combining the results for radiative transfer in starburst galaxies for what we now know is a more varied story of dust extinction curves in the Milky Way and Magellanic Clouds, it is most probable that the dust grains in starburst galaxies are truly lacking the $2175 \AA$ feature and possesses strong far-ultraviolet extinctions. It is very important to clearly state that this conclusion only applies to the dust found in or near the starburst regions. This is the dust which is probed by the ultraviolet photons from the starburst regions. Given our knowledge of Local Group dust extinction curves, it is very likely that dust far from sites of active star formation in starburst galaxies would be characterized by Milky Way-like or quiescent dust (i.e., Cardelli et al. [7] relationship).

The measurements of starburst galaxies in the ultraviolet necessarily probe only the active regions of these galaxies; there are other measurements which probe the type of dust found in more quiescent regions of galaxies. Studies of the colors of gravitational lens systems [31, 32] have found Cardelli et al. [7] type dust curves. As gravitational lenses probe random sightlines in galaxies, this is evidence that quiescent dust is like that seen in our Galaxy. These gravitational lens difference curves are not direct measures of dust extinction as they probe the difference in two dust extinction curves of unknown dust columns [33, 34], but they can be used to determine the presence of the $2175 \AA$ feature. Finally, measurements of $\mathrm{Mg}$ II absorbers also probe random sightlines in galaxies and show evidence for the $2175 \AA$ feature [35, 36]. 


\section{ACKNOWLEDGMENTS}

I would like thank the organizers of this conference for inviting me to give a talk on my work. The work described in the paper encompasses a large body of work, which would not have been possible but for the contributions of many of my collaborators. I would like to especially thank Adolf Witt who was my Monte Carlo radiative transfer mentor and Geoff Clayton who was my extinction curve mentor.

\section{REFERENCES}

1. Gordon, K. D., Misselt, K. A., Witt, A. N., and Clayton, G. C., ApJ, 551, 269 (2001).

2. Misselt, K. A., Gordon, K. D., Clayton, G. C., and Wolff, M. J., ApJ, 551, 277 (2001).

3. Weingartner, J. C., and Draine, B. T., ApJ, 548, 296 (2001).

4. Clayton, G. C., Wolff, M. J., Sofia, U. J., Gordon, K. D., and Misselt, K. A., ApJ, 588, 871 (2003).

5. Zubko, V., Dwek, E., and Arendt, R. G., ApJS, 152, 211 (2004).

6. Gordon, K. D., "Interstellar Dust Scattering Properties," in ASP Conf. Ser. 309: Astrophysics of Dust, 2004, p. 77.

7. Cardelli, J. A., Clayton, G. C., and Mathis, J. S., ApJ, 345, 245 (1989).

8. Fitzpatrick, E. L., and Massa, D., ApJS, 72, 163 (1990).

9. Gordon, K. D., Clayton, G. C., Misselt, K. A., Landolt, A. U., and Wolff, M. J., ApJ, 594, 279 (2003).

10. Calzetti, D., Kinney, A. L., and Storchi-Bergmann, T., ApJ, 429, 582 (1994).

11. Calzetti, D., AJ, 113, 162 (1997).

12. Prevot, M. L., Lequeux, J., Prevot, L., Maurice, E., and Rocca-Volmerange, B., A\&A, 132, 389 (1984).

13. Witt, A. N., and Gordon, K. D., ApJ, 463, 681 (1996).

14. Gordon, K. D., Calzetti, D., and Witt, A. N., ApJ, 487, 625 (1997).

15. Gordon, K. D., Smith, T. L., and Clayton, G. C., "Dust in High Redshift Starburst Galaxies," in ASP Conf. Ser. 193: The Hy-Redshift Universe: Galaxy Formation and Evolution at High Redshift, 1999, p. 517.

16. Vijh, U. P., Witt, A. N., and Gordon, K. D., ApJ, 587, 533 (2003).

17. Witt, A. N., and Gordon, K. D., ApJ, 528, 799 (2000).

18. Granato, G. L., Silva, L., Bressan, A., Lacey, C. G., Baugh, C. M., Cole, S., and Frenk, C. S., Astrophysics and Space Science Supplement, 277, 79 (2001).

19. Fischera, J., Dopita, M. A., and Sutherland, R. S., ApJL, 599, L21 (2003).

20. Pierini, D., Gordon, K. D., Witt, A. N., and Madsen, G. J., ApJ, in press (2004).

21. Clayton, G. C., and Martin, P. G., ApJ, 288, 558 (1985).

22. Fitzpatrick, E. L., AJ, 92, 1068 (1986).

23. Gordon, K. D., and Clayton, G. C., ApJ, 500, 816 (1998).

24. Lequeux, J., Maurice, E., Prevot-Burnichon, M.-L., Prevot, L., and Rocca-Volmerange, B., A\&A, 113, L15 (1982).

25. Misselt, K. A., Clayton, G. C., and Gordon, K. D., ApJ, 515, 128 (1999).

26. Valencic, L. A., Clayton, G. C., and Gordon, K. D., ApJ, 616, 912 (2004).

27. Valencic, L. A., Clayton, G. C., Gordon, K. D., and Smith, T. L., ApJ, 598, 369 (2003).

28. Clayton, G. C., Gordon, K. D., and Wolff, M. J., ApJS, 129, 147 (2000).

29. Clayton, G. C., Gordon, K. D., Salama, F., Allamandola, L. J., Martin, P. G., Snow, T. P., Whittet, D. C. B., Witt, A. N., and Wolff, M. J., ApJ, 592, 947 (2003).

30. Whittet, D. C. B., Shenoy, S. S., Clayton, G. C., and Gordon, K. D., ApJ, 602, 291 (2004).

31. Nadeau, D., Yee, H. K. C., Forrest, W. J., Garnett, J. D., Ninkov, Z., and Pipher, J. L., ApJ, 376, 430 (1991).

32. Falco, E. E., Impey, C. D., Kochanek, C. S., Lehár, J., McLeod, B. A., Rix, H.-W., Keeton, C. R., Muñoz, J. A., and Peng, C. Y., ApJ, 523, 617 (1999).

33. Wucknitz, O., Wisotzki, L., Lopez, S., and Gregg, M. D., A\&A, 405, 445 (2003).

34. McGough, C., Clayton, G. C., Gordon, K. D., and Wolff, M. J., ApJ, in press (2005). 
35. Malhotra, S., ApJL, 488, L101 (1997).

36. Wang, J., Hall, P. B., Ge, J., Li, A., and Schneider, D. P., ApJ, 609, 589 (2004). 